\title{
Numerical techniques for linear and nonlinear eigenvalue problems in the theory of elasticity
}

\author{
Aliki D. Muradova*
}

(Received 19 November 2004, revised 12 April 2005)

\begin{abstract}
Consider algorithms to solve eigenvalue problems for partial differential equations describing the bending of a von Kármán elastic plate. Here we explore numerical techniques based on a variational principle, Newton's iterations and numerical continuation. The variational approach uses the Galerkin spectral method. First we study the linearized problem. Second, eigenfunctions of the nonlinear equations describing post-buckling behaviour of the von Kármán plate are calculated. The plate is supposed to be totally clamped and compressed along its four sides. The basis functions in the variational procedure are trigonometric functions. They are chosen to match the boundary conditions. Effective computational techniques allow us to detect bifurcation points and to trace branches of solutions. Numerical examples demonstrate the efficiency of the methods. The proposed
\end{abstract}

*The Mathematical Sciences Institute, The Australian National University, Canberra, AustraliA. mailto:Aliki.Muradova@maths.anu.edu.au

See http://anziamj.austms.org.au/V46/CTAC2004/Mura for this article, (C) Austral. Mathematical Soc. 2005. Published May 24, 2005. ISSN 1446-8735 
algorithms are applicable to similar problems involving elliptic differential equations.

\section{Contents}

1 Introduction

2 Mechanical formulation

C428

3 Eigenvalues of the linearized problem

C430

4 Spectral method and continuation for nonlinear problems C432

5 Numerical examples

C437

References

C437

\section{Introduction}

I aim to demonstrate robust numerical techniques for a class of problems which describe the behaviour of thin elastic rectangular plates. The basic idea lies in variational studies of mechanical models. We will find eigenvalues and eigenfunctions using the Galerkin spectral method.

Post-buckling behaviour of the von Kármán plate has been investigated by several authors and there are many numerical approaches for treating these mechanical models. The majority of the numerical methods employ either finite elements or finite differences. The existing techniques for discrete schemes are based on the Newton-GMRES algorithm [2, 5, e.g.] and sophisticated numerical continuation [1]. For example, Chien, Chang \& Mei [2] applied the GMRES algorithm in the context of numerical continuation for 
the plate compressed on its two sides. The most general case of bifurcation phenomenon was studied numerically by Allgower \& Georg [1]. Holder, Schaeffer [7] and Golubitsky [8] have shown that so-called mode jumping, when the primary solution branches lose stability through further bifurcation "may occur under the partially but not for the simply supported conditions". Mode jumping may also occur under totally clamped conditions. Dossou \& Pierre [5] recently analyzed deformation and bifurcation for the discrete von Kármán problem by the Newton-GMRES approach.

Here we propose a spectral method with a choice of global trial functions which span the whole domain. This approach guarantees high accuracy and computational efficiency. In Section 2 we formulate the nonlinear mechanical model and consider the totally clamped (Dirichlet and Neumann) conditions. The plate is subjected to a uniform lateral compression on its four sides. The totally clamped conditions are the most interesting, since then the behaviour of the solution becomes more complicated. Section 3 is devoted to a numerical analysis of the linearized von Kármán problem. We use the Galerkin procedure to compute eigenvalues. Section 4 demonstrates the spectral method for the nonlinear model and the numerical continuation algorithm for tracing the branches of the solutions. Some numerical examples are illustrated in Section 5.

\section{Mechanical formulation}

The two-dimensional model of bending and stretching of a rectangular plate with buckling is

$$
\begin{aligned}
D \Delta^{2} w & =2 h_{0}[w, \psi]+\lambda[\theta, w], \quad(x, y) \in G, \\
\Delta^{2} \psi & =-\frac{E}{2}[w, w]
\end{aligned}
$$


where $G=\left(0, l_{1}\right) \times\left(0, l_{2}\right)$ is the shape of the plate $\left(l_{1}\right.$ and $l_{2}$ are lengths of the sides of the plate),

$$
[w, \psi]=\frac{\partial^{2} w}{\partial x^{2}} \frac{\partial^{2} \psi}{\partial y^{2}}+\frac{\partial^{2} \psi}{\partial x^{2}} \frac{\partial^{2} w}{\partial y^{2}}-2 \frac{\partial^{2} w}{\partial x \partial y} \frac{\partial^{2} \psi}{\partial x \partial y},
$$

$w(x, y)$ denotes the deflection, $\psi(x, y)$ is the Airy stress potential, $\theta$ is a regular function defined on $G$, with values which depend on the portion of the boundary, subjected to compression, $\lambda$ is the intensity of this compression, $D=2 E h_{0}^{3} /\left[3\left(1-\nu^{2}\right)\right]$ is the cylindrical rigidity, $E$ is Young's modulus, $2 h_{0}$ is a thickness of the plate and $\nu$ is Poisson's ratio. If the plate is compressed on each side then

$$
\theta(x, y)=-\frac{1}{2}\left(x^{2}+y^{2}\right)
$$

I assume that all physical parameters in (1) have unit magnitude (the techniques are applicable for different values of $\left.D, h_{0}, E\right)$. This assumption is valid for polygonal plates.

The plate, compressed on its two ends was considered by [8, 7, 3, 2, e.g.]. We study the case in which the plate is compressed on its four sides, that is, (2) holds. Thus, (1) becomes

$$
\begin{aligned}
\Delta^{2} w & =[w, \psi]-\lambda \Delta w, \quad(x, y) \in G, \\
\Delta^{2} \psi & =-[w, w] .
\end{aligned}
$$

The linearized (spectral) equation implies

$$
\Delta^{2} w+\lambda \Delta w=0, \quad(x, y) \in G .
$$

If the plate is totally clamped, the classical boundary conditions on the edges are

$$
\begin{array}{ll}
w=\frac{\partial w}{\partial n}=0, \quad(x, y) \in \partial G, \\
\psi=\frac{\partial \psi}{\partial n}=0, \quad(x, y) \in \partial G .
\end{array}
$$


Here $\frac{\partial}{\partial n}$ denotes the normal derivative. According to the results of [4], if $\lambda \leq$ $\lambda_{11}$, where $\lambda_{11}$ is the first eigenvalue of the linearized problem (4) and (5 $)$ then (3) and (5) has a unique trivial solution. If $\lambda>\lambda_{11}$ then (3) and (5) has at least three solutions: $(w, \psi),(-w, \psi),(w \neq 0, \psi \neq 0)$ and $(0,0)$.

\section{Eigenvalues of the linearized problem}

The first step of the numerical analysis is to detect primary bifurcation points. Actually, buckling loads $\lambda$, for which the solution of (3) and (5) bifurcates from the trivial one correspond to eigenvalues of the appropriated linearized problem (4) and (51). The corresponding branches we call primary branches and subsequent bifurcations from them are called secondary ones. The secondary bifurcation points can be detected by a Newton-based method with rationally computed quantities, suggested by Harrar \& Osborne [6]. This idea is also described in [5]. Singular points are detected along solution curves by a scalar quantity, called a detector. The secondary bifurcation often results from splitting a double eigenvalue by perturbation.

To compute eigenvalues of the linearized problem (4) and (5 $)$ the Galerkin procedure is applied. The solution is found as partial sums of double series

$$
W_{N}(x, y)=\sum_{i, j=1}^{N} w_{N}^{i j} \omega_{i j}(x, y),
$$

where $\omega_{i j}(x, y)$ are global trial functions and $N$ is a natural number.

The coordinate functions $\omega_{i j}(x, y)=\chi^{2} P_{i}(x) \chi^{2} P_{j}(y)$, where the second order divided differences with respect to the indexes of the Legendre polynomials

$$
\chi^{2} P_{i}(x)=\sqrt{\frac{2 i+1}{2}}\left(a_{i} P_{i+2}(x)+c_{i} P_{i}(x)+b_{i} P_{i-2}(x)\right)
$$




$$
\text { with } \quad \begin{aligned}
a_{i} & =\frac{1}{(2 i+1)(2 i+3)}, \\
c_{i} & =-\frac{2}{(2 i-1)(2 i+3)}, \\
b_{i} & =\frac{1}{(2 i-1)(2 i+1)} .
\end{aligned}
$$

Vashakmadze [9] applied these to similar linear problems on the square $\bar{G}=$ $[-1,1]^{2}$. We use them for (4) and $\left(5_{1}\right)$.

For our purposes it is more convenient to use combinations of trigonometric functions for solving the nonlinear equations. They reflect the shape of the eigenfunctions of the nonlinear problem and the Fourier coefficients of the expansion are good guides for following branches of the solutions. Therefore, we introduce a new basis for (4) and $\left(5_{1}\right)$, which will be applied to the nonlinear problem as well. The basis is

$$
\omega_{i j}(x, y)=\chi \cos \left(\frac{i}{l_{1}} \pi x\right) \chi \cos \left(\frac{j}{l_{2}} \pi y\right),
$$

where $\chi \cos \left(\frac{i}{l} \pi x\right)=\sqrt{2 / l}\left(\cos \left(\frac{i+1}{l} \pi x\right)-\cos \left(\frac{i-1}{l} \pi x\right)\right)$ and $\left\{\chi \cos \left(\frac{i}{l} \pi x\right)\right\}_{i=1}^{N}$ is a system of linear independent elements for arbitrary $N$, complete in the Sobolev space $W^{2,2}(G)$ and satisfying the boundary conditions $\left(5_{1}\right)^{1}$.

The Galerkin method gives the linear discrete eigenvalue problem

$$
\left(K w_{N}\right)_{m n}=\lambda\left(B w_{N}\right)_{m n}, \quad m, n=1,2, \ldots, N,
$$

where $\left(K w_{N}\right)_{m n}=\left\langle\Delta^{2} W_{N}, \omega_{m n}\right\rangle$ and $\left(B w_{N}\right)_{m n}=-\left\langle\Delta W_{N}, \omega_{m n}\right\rangle$. The scalar products are taken in $L_{2}$. The system (8) is split into four subsystems, subjected to evenness of indexes $(m, n)$. Each of these subsystems is

${ }^{1}$ Note, $\cos \left(\frac{i}{l} \pi x\right)-\cos \left(\frac{i+2}{l} \pi x\right)$ are eigenfunctions of the ordinary differential equation which is obtained after the use of the rule of separation of variables to $\Delta^{2} w+\lambda w_{x x}=0$ $[8,7,2$, e.g.]. The boundary conditions are partially clamped. 
solved separately. Under $l_{1}=l_{2}=1, N=30$ the computed eigenvalues $\lambda_{m n}$ $(m, n=1,2, \ldots, 5)$ are:

$\begin{array}{llllllll}52.345 & 92.126 & 92.126 & 128.215 & 154.130 & 167.031 & 189.583 & 189.583\end{array}$ $\begin{array}{lllllllll}246.327 & 246.327 & 246.351 & 269.141 & 279.092 & 326.681 & 326.681 & 349.327\end{array}$ $\begin{array}{llllllll}362.755 & 380.333 & 380.333 & 403.927 & 425.485 & 434.837 & 481.699 & 481.699\end{array}$ 501.061

\section{The spectral method and numerical continuation for the nonlinear problem}

The techniques, introduced in Section 3, are extended to (3) and (5). Thus,

$$
W_{N}(x, y)=\sum_{i, j=1}^{N} w_{N}^{i j} \omega_{i j}(x, y), \quad \Psi_{N}(x, y)=\sum_{i, j=1}^{N} \psi_{N}^{i j} \varphi_{i j}(x, y),
$$

where $\omega_{i j}(x, y)=\varphi_{i j}(x, y)=\chi \cos \left(\frac{i}{l_{1}} \pi x\right) \chi \cos \left(\frac{j}{l_{2}} \pi y\right)$, see (7). After applying the Galerkin method to (3) and (5) we obtain the nonlinear algebraic equations

$$
\begin{aligned}
& \left(K_{1} w_{N}\right)_{m n}=A_{1, N}^{m n}\left(w_{N}, \psi_{N}\right)+\lambda\left(B w_{N}\right)_{m n}, \\
& \left(K_{2} \psi_{N}\right)_{m n}=A_{2, N}^{m n}\left(w_{N}, w_{N}\right), \quad m, n=1,2, \ldots, N .
\end{aligned}
$$

Here $K_{1, N}, K_{2, N}$ and $B_{N}$ are linear and $A_{1, N}$ and $A_{2, N}$ are nonlinear discrete operators.

We solve (10) by a combination of the Newton and numerical continuation algorithms with respect to the load parameter $\lambda$. An alternative is to fix $\lambda$ and vary the plate dimensions.

Let $\eta$ be an eigenvalue of the linearized problem (4) and $\left(5_{1}\right)$. Consider $\lambda \in\left[\lambda_{1}, \lambda_{1}+\Lambda\right]$, where $\lambda_{1}=\eta+\varepsilon, \varepsilon$ is a small positive number and $\Lambda$ is 
an arbitrary positive number. Suppose $\lambda_{k+1}=\lambda_{k}+\delta_{k}, k=1,2, \ldots, M-1$, $\lambda_{M}=\lambda_{1}+\Lambda$. To start Newton's iterations for (10) we choose $\lambda=\lambda_{1}$, then use the previous results for the next $\lambda_{2}$ and continue so on.

Below we demonstrate an effective algorithm for tracing the branches of the eigenfunctions. It computes the coefficients $w_{N}^{i j}$ in (9).

1. Start with $\lambda=\lambda_{k}=\eta+\varepsilon$, where $k=1$.

2. Choose the initial approximations $w_{N}^{i j}\left(\lambda_{k}\right)$ for the coefficients $w_{N}^{i j}\left(\lambda_{k}\right)$ in the expansion (9) putting $w_{N}^{i_{1} j_{1}}\left(\lambda_{k}\right)=c(\varepsilon) \neq 0, w_{N}^{i j}\left(\lambda_{k}\right)=0, i \neq i_{1}$, $j \neq j_{1}$. Here $w_{N}^{i_{1}, j_{1}}$ is the largest coefficient in the expansion (9).

3. Solve (10) by the Newton method with the initial approximations which have been chosen at Step 2. If the iterative process approaches the trivial solution or diverges then increase or decrease $|c(\varepsilon)|$ respectively and return to Step 2.

4. Put $\lambda=\lambda_{k+1}=\lambda_{k}+\delta_{k}$, where $\delta_{k}$ is a positive number.

5. Choose the initial approximations $w_{N}^{i j}\left(\lambda_{k+1}\right)$ putting $w_{N}^{i j}\left(\lambda_{k+1}\right)=w_{N}^{i j}\left(\lambda_{k}\right)$, $i, j=1,2, \ldots, N$, where $w_{N}^{i j}\left(\lambda_{k}\right)$ were computed before.

6. Solve (10) by the Newton method with the initial approximations which have been chosen at Step 5. If the process diverges or approaches the trivial solution then decrease $\delta_{k}$ and return to Step 4.

7. Repeat Steps 4-6 for $k=2,3 \ldots, M-1$ until $\lambda=\lambda_{M}=\lambda_{1}+\Lambda$.

In the algorithm the largest coefficient $w_{N}^{i_{1} j_{1}}$ is revealed by solving the linearized problem (4) and (51). For instance, for $\eta=\lambda_{11}=52.345$ (see the example in Section 3) the largest component of the corresponding eigenvector 


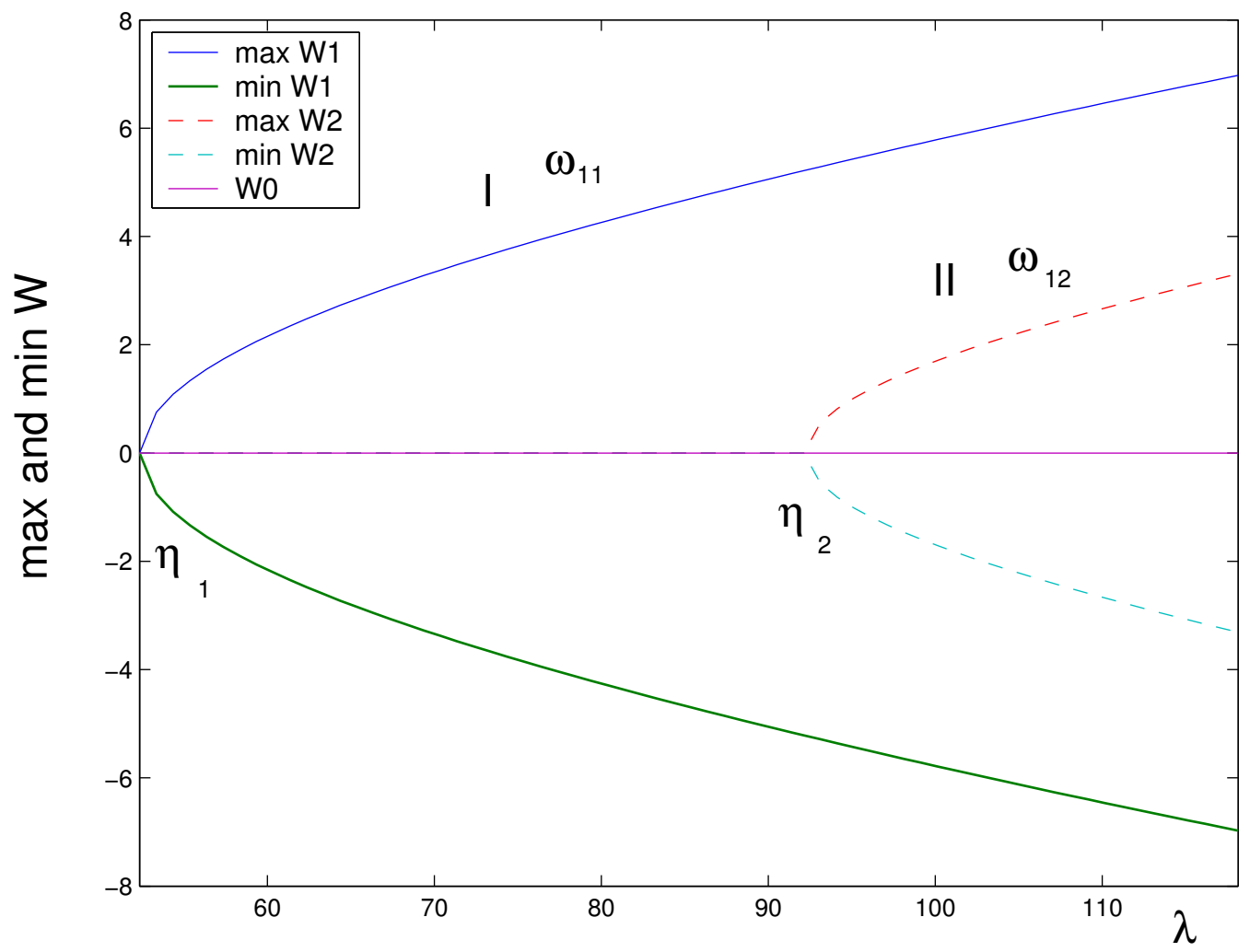

Figure 1: $l_{1}=l_{2}=1, N=6, \eta_{1}=\lambda_{11}=52.345, \eta_{2}=\lambda_{12}=\lambda_{21}=92.126$.

is $w_{N}^{11}$. Therefore in the algorithm we should put $\stackrel{(0)}{w_{N}^{11}}\left(\lambda_{k}\right)=c(\varepsilon) \neq 0$ (for example, $c=0.1, \varepsilon=1, N=5$ ).

If the solution jumps on the other branch at Step 6 then we deal with the secondary bifurcation or mode jumping.

The methods have been implemented in Python. Numerical examples are demonstrated in Section 5. 


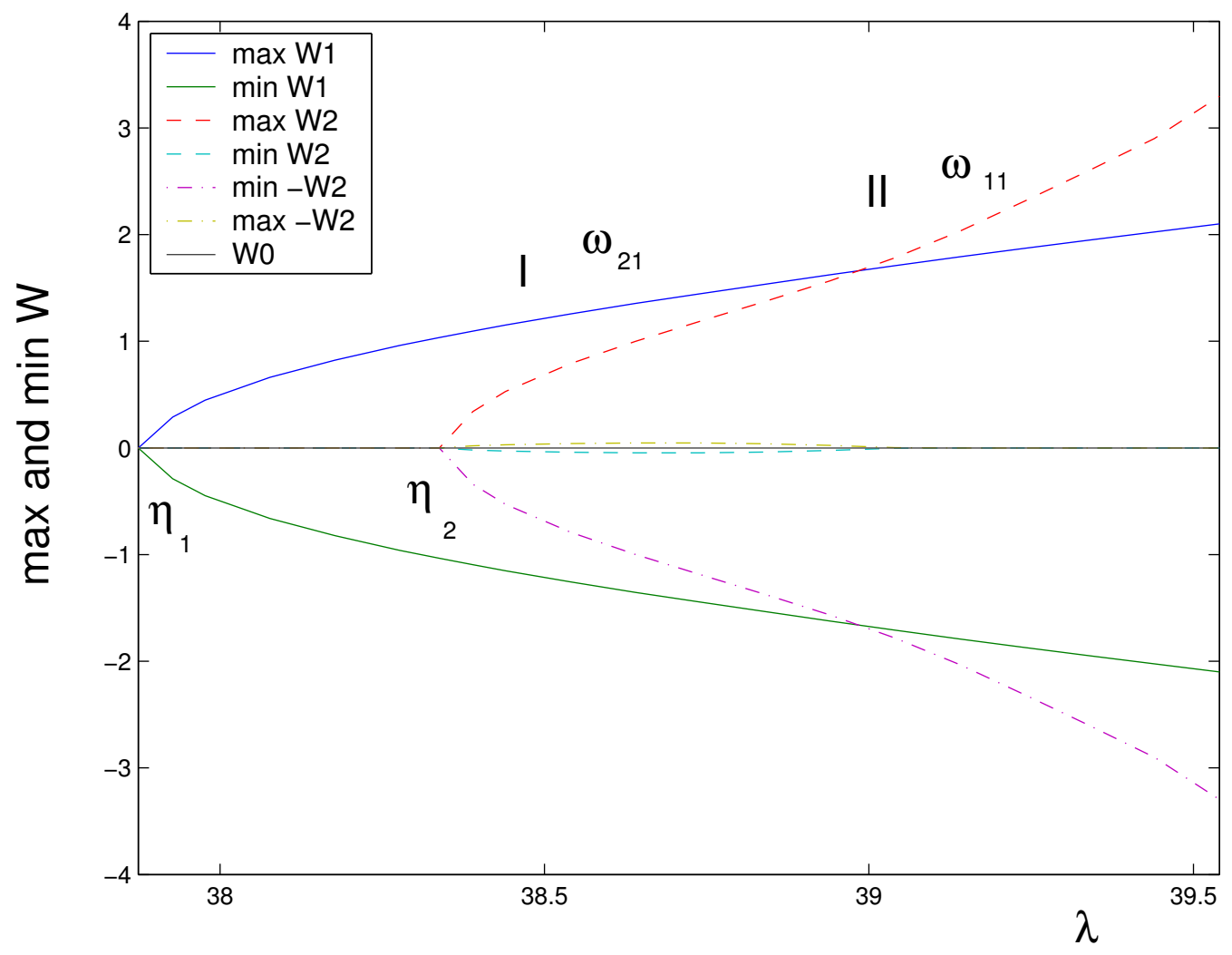

Figure $2: l_{1}=3.3, l_{2}=1, N=6, \eta_{1}=\lambda_{21}=37.874, \eta_{2}=\lambda_{11}=38.338$. 


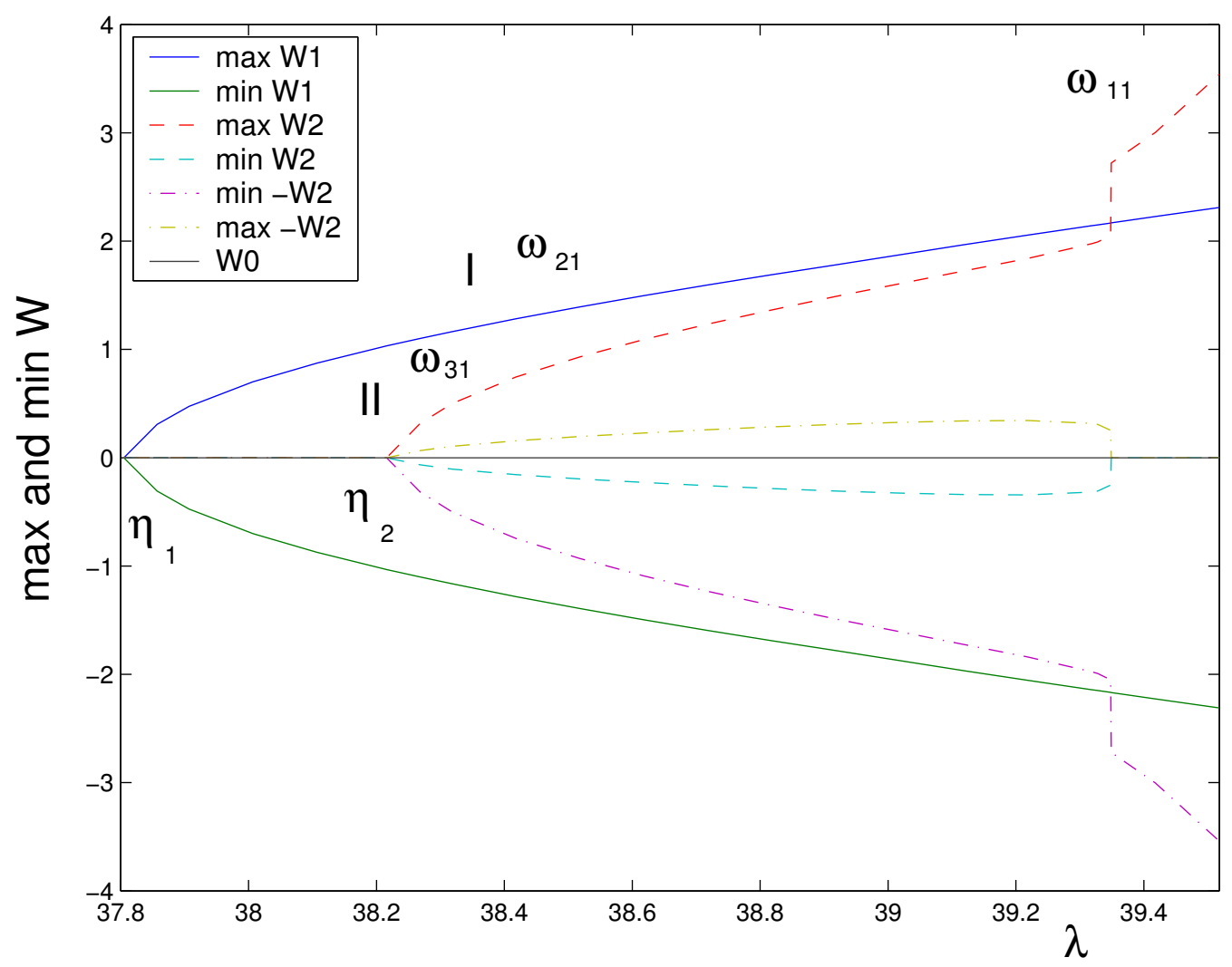

Figure $3: l_{1}=3.5, l_{2}=1, N=6, \eta_{1}=\lambda_{21}=37.805, \eta_{2}=\lambda_{31}=38.216$. 


\section{$5 \quad$ Numerical examples}

Figures 1, 2 and 3 show $\max _{G_{M}} W_{N}\left(x_{i}, y_{j}\right)$ and $\min _{G_{M}} W_{N}\left(x_{i}, y_{j}\right)\left(G_{M}=\right.$ $\left.\left\{\left(x_{i}, y_{j}\right) \mid i, j=1,2, \ldots, 10\right\}\right)$ of the first two curves of the solution $W_{N}$ (Branches I and II) and their symmetrical ones with different sizes of the plate. In Figures 1, 2 and $3, \omega_{i j}$ denotes that trial function which has the largest coefficient in the expansion (9). On Figure 1 Branch I has one nodal domain, Branch II has two nodal domains in which $\max _{G_{M}} W_{N}=$ $-\min _{G_{M}} W_{N}$ and $\eta_{2}$ is a double bifurcation point. On Figure 2 Branch I has two nodal domains and $\max _{G_{M}} W_{N}=-\min _{G_{M}} W_{N}$, Branch II has three nodal domains from the beginning, in which $\max _{G_{M}} W_{N} \neq-\min _{G_{M}} W_{N}$, then it changes the configuration at the point $\lambda=39.090$ (secondary bifurcation point) and has only one nodal domain. On Figure 3 Branch I has two nodal domains and $\max _{G_{M}} W_{N}=-\min _{G_{M}} W_{N}$, Branch II has three nodal domains from the beginning, in which $\max _{G_{M}} W_{N} \neq-\min _{G_{M}} W_{N}$, then it changes the configuration at $\lambda=39.349$ (secondary bifurcation point) and has only one nodal domain.

Acknowledgment: I am grateful to Prof. Mike Osborne (ANU) for discussions and useful suggestions; Dr. Kokou Dossou (UTS) who helped to improve my understanding of the subject, and Assoc. Prof. George Stavroulakis (UOI) and Prof. Tamaz Vashakmadze (TSU) for their interest in this work.

\section{References}

[1] E. L. Allgower and K. Georg. Numerical continuation methods. Springer, Berlin, 1990. C427, C428

[2] C. S. Chien, S. L. Chang and Z. Mei. Tracing the buckling of a rectangular plate with the Block GMRES method. J. of Comput. and 
Appl. Math., 136, pages 199-218, 2001.

http://dx.doi.org/10.1016/S0377-0427(00)00611-7. C427, C429, C431

[3] C. S. Chien, S. Y. Gong and Z. Mei. Mode jumping in the von Kármán equations. SIAM J. Sci. Comput., volume 22, No 4, pages 1354-1385, 2000. [Online] http://epubs.siam.org/sam-bin/dbq/article/30732. C429

[4] P. Ciarlet and P. Rabier. Les equations de von Kármán. Springer-Verlag, Berlin, Heidelberg, New York, 1980. C430

[5] K. Dossou and R. Pierre. A Newton-GMRES approach for the analysis of the postbuckling behavior of the solutions of the von Kármán equations. SIAM J. Sci. Comput., volume 24, No 6, pages 1994-2012, 2003. [Online] http://epubs.siam.org/sam-bin/dbq/article/37614. C427, C428, C430

[6] D. L. Harrar and M. R. Osborne. Computing eigenvalues of ordinary differential equations. ANZIAM J., 44(E), pages C313-C334, 2003. [Online] http://anziamj.austms.org.au/V44/CTAC2001/Harr. C430

[7] E. J. Holder and D. G. Schaeffer. Boundary conditions and mode jumping in the von Kármán's equations. SIAM, J. Math. Anal., 15, pages 446-458, 1984.

http://locus.siam.org/SIMA/volume-15/art_0515034.html. C428, C429, C431

[8] D. G. Schaeffer and M. Golubitsky. Boundary conditions and mode jumping in the buckling of a rectangular plate. Comm. Math. Phys., 69, pages 209-236, 1979. URL C428, C429, C431

[9] T. S. Vashakmadze. The theory of anisotropic elastic plates. Kluwer Academic Publishers, Dortrecht, Boston, London, 1999. C431 